\title{
Reducing tuberculosis transmission: a consensus document from the World Health Organization Regional Office for Europe
}

Giovanni Battista Migliori (1) ${ }^{1,8}$, Edward Nardell 2,8, Askar Yedilbayev $^{3}$, Lia D’Ambrosio (104 ${ }^{4}$, Rosella Centis (1) ${ }^{1}$, Marina Tadolini ${ }^{5}$, Martin van den Boom ${ }^{6,8}$, Soudeh Ehsani ${ }^{6}$, Giovanni Sotgiu ${ }^{7,8}$ and Masoud Dara ${ }^{6}$

Affiliations: ${ }^{1}$ Respiratory Diseases Clinical Epidemiology Unit, Clinical Scientific Institutes Maugeri, IRCCS, Tradate, Italy. ${ }^{2}$ Division of Global Health Equity, Harvard Medical School, Brigham and Women's Hospital, Boston, MA, USA. ${ }^{3}$ Partners In Health, Boston, MA, USA. ${ }^{4}$ Public Health Consulting Group, Lugano, Switzerland. ${ }^{5}$ Dept of Medical and Surgical Sciences, Alma Mater Studiorum University of Bologna, Bologna, Italy. ${ }^{6}$ Joint Tuberculosis, HIV and Viral Hepatitis Programme, WHO Regional Office for Europe, Copenhagen, Denmark. ${ }^{7}$ Clinical Epidemiology and Medical Statistics Unit, Dept of Medical, Surgical and Experimental Sciences, University of Sassari, Sassari, Italy. ${ }^{8}$ These authors contributed equally to this work.

Correspondence: Giovanni Battista Migliori, Istituti Clinici Scientifici Maugeri, IRCCS, Via Roncaccio 16, 21049 Tradate, Italy. E-mail: giovannibattista.migliori@icsmaugeri.it

@ERSpublications

Sound implementation of administrative and environmental controls, personal protection and FAST approach (rapid diagnosis and effective treatment tailored to drug resistance profile with focus on undetected cases) are necessary to reduce TB transmission http://ow.ly/hEhw30of6Gk

Cite this article as: Migliori GB, Nardell E, Yedilbayev A, et al. Reducing tuberculosis transmission: a consensus document from the World Health Organization Regional Office for Europe. Eur Respir J 2019; 53: 1900391 [https://doi.org/10.1183/13993003.00391-2019].

ABSTRACT Evidence-based guidance is needed on 1) how tuberculosis (TB) infectiousness evolves in response to effective treatment and 2) how the TB infection risk can be minimised to help countries to implement community-based, outpatient-based care.

This document aims to 1) review the available evidence on how quickly TB infectiousness responds to effective treatment (and which factors can lower or boost infectiousness), 2) review policy options on the infectiousness of TB patients relevant to the World Health Organization European Region, 3) define limitations of the available evidence and 4) provide recommendations for further research.

The consensus document aims to target all professionals dealing with TB (e.g. TB specialists, pulmonologists, infectious disease specialists, primary healthcare professionals, and other clinical and public health professionals), as well as health staff working in settings where TB infection is prevalent. 


\section{Introduction}

Ongoing transmission, infection and re-infection, in both community and congregate settings, are fuelling the global tuberculosis (TB) epidemic [1]. Although traditional TB transmission control efforts have focused mainly on known TB patients, evidence exists that TB patients on effective therapy become rapidly noninfectious and that unsuspected, untreated TB cases (and unsuspected drug resistance) account for most transmission [1]. In the Former Soviet Union (FSU) countries a combination of prolonged hospitalisation, delayed diagnosis of drug resistance and poor ventilation has resulted in "hyper-transmission", with a prevalence of multidrug-resistant (MDR)-TB of $\geqslant 25 \%$ among new cases, compared with $<7 \%$ in the majority of the other settings [1-4].

More evidence-based guidance is needed on 1) how quickly TB infectiousness responds to effective treatment and 2) how the TB infection risk can be minimised. This will help to move away from traditional hospital-based treatment and care towards more community/outpatient-based care, in an effort to ultimately contribute to reducing TB-related suffering and improve TB treatment outcomes.

This document aims to review and discuss the available evidence based on five crucial questions:

1. Which patients are infectious and which factors favour disease transmission?

2. When does an infectious patient become noninfectious after starting treatment?

3. What can render the patient noninfectious more or less rapidly?

4. Which patients need hospital admission (and respiratory isolation) because of their infectiousness?

5. What are the research needs?

\section{Methods}

Owing to the difficulty of summarising the five questions within an overarching research question, it was necessary to use a research methodology incorporating a wide search of scientific manuscripts using different search engines. It was particularly important to assess the current grey literature, along with studies preceding the internet era.

Relevant scientific documents published in English (in Google Scholar and other grey literature sources) were identified using the Google search engine and the following key words: "tuberculosis", "MDR-TB" (including extensively drug-resistant (XDR)-TB), "infectiousness", "contagiousness", "transmission" and "infection control". As a systematic review covering these topics was published in 2013 [5], the search focused on the 2013-2017 period. Historical articles were retrieved from World Health Organization (WHO) and International Union Against Tuberculosis and Lung Disease documents [6, 7]. A nonsystematic approach was adopted because of the short period since the last published systematic review and the large time span from historical studies to the more recent ones.

All retrieved documents were evaluated, together with the references in the main articles, in order to answer the questions as fully as possible.

\section{Probability of TB transmission}

$\mathrm{TB}$ infection is a probabilistic phenomenon, involving many different factors $[1,2,6]$. For TB infection to occur, at least one inhaled viable and virulent Mycobacterium tuberculosis droplet nucleus must reach the alveolar macrophages within the lung. The host needs to inhale multiple droplet nuclei during either a single exposure or repeated exposures to become infected. The source strength (number of droplet nuclei generated) is therefore an important factor: this can be influenced by factors such as cough strength and frequency, presence of lung cavities, sputum viscosity, and ventilation. "Hyper-transmitters" (i.e. highly infectious, undiagnosed cases) probably account for most recognised TB outbreaks [1, 2]. Features of the host such as immunosuppression and comorbidities are also very important. To minimise exposure, it is extremely important that the index case should receive effective treatment so as to rapidly become noninfectious.

\section{Current WHO guidance on TB infection control}

Managerial activities are necessary to support infection control (table 1) on top of administrative controls, environmental controls and personal protection [8]. All of the main guidelines in force follow this approach [9-13]. The focus, however, is often the known TB patient (usually promptly placed on therapy, thereby ending transmission), whereas unsuspected cases (or drug resistance) are neglected and thus contribute to transmission in congregate settings. The principles of a refocused, intensified, administrative TB transmission control strategy, known as FAST (Find cases Actively by cough surveillance and rapid molecular sputum testing, Separate safely, and Treat effectively based on rapid drug susceptibility testing (DST)), have also been used in developing this document [14, 15]. The pilot experience in different countries (including Bangladesh, Nigeria, Peru, the Russian Federation and Vietnam) showed a high 
TABLE 1 Measures for tuberculosis (TB) infection control in healthcare settings: managerial activities

1. Identify and strengthen a coordinating body for TB infection control, and develop a comprehensive budgeted plan that includes human resource requirements for implementing TB infection control at all levels

2. Ensure the appropriate design, construction, renovation, maintenance and use of health facilities

3. Conduct TB surveillance among health workers and assessment at all levels of the health system and in congregate settings

4. Address TB infection control advocacy, communication and social mobilisation, including engagement of civil society

5. Monitor and evaluate the set of TB infection control measures

6. Enable and conduct operational research

Adapted from [8].

proportion of undetected TB patients (or undetected drug resistance with regimen not adapted to the resistance profile) among hospitalised cases: detection and treatment were improved by implementing the FAST approach $[1,2,16,17]$.

\section{Administrative controls}

Administrative controls are policies and work practices aimed at reducing the risk of exposure, infection and disease (summarised in table 2).

\section{Environmental controls}

Environmental controls are equipment or practices aimed at reducing the concentration of infectious bacilli in the air in areas where contamination is likely (summarised in table 3 ).

The importance of ventilation and air circulation was demonstrated following a TB outbreak on a US navy ship $[18,19]$. The study found that living in the same compartment with an infectious TB patient or sharing air from a closed-circuit ventilation system was the core determinant of TB transmission. There was no evidence for significant transmission from any other form of contact aboard the ship, such as contact with fomites or casual contact in different areas.

It is not possible to culture human-generated M. tuberculosis strains from ambient room air. Molecular detection methods have so far proven to be able to estimate the number of bacilli in air, but not measure their viability and infectivity [20]. The time from droplet nuclei generation to infection can be brief (a few seconds). Two laboratory studies have evaluated M. tuberculosis survival time (half-life) in a Goldberg drum (used for aerosol survival studies with artificially aerosolised organisms, not naturally generated aerosol from patients), with differing results: $6 \mathrm{~h}$ in the Loudon study of 1969 [21, 22] and 20 min in the Lever study of 2003 [23]. It is probable that survival also depends on local conditions, temperature and humidity, for example, and varies greatly. It is important to note that once settled, it is nearly impossible to re-aerosolise droplets with sweeping, shaking out bedclothes and other activities that sometimes cause concern. Re-suspended particles are generally far too large to reach their target alveolar macrophage cells in the distal lung.

The size of the expelled droplets and their velocity (affected by sneezing, breathing or coughing) is crucial to influence evaporation and distance reached; however, only droplet nuclei transmit TB.

The importance of outside air ventilation (natural or mechanical) in protecting healthcare workers (HCWs) has been reported [24-28]. Outside air ventilation can be estimated as air changes per hour

TABLE 2 Measures for tuberculosis (TB) infection control in healthcare settings: administrative controls

1. Promptly identify people with TB symptoms (triage), separate infectious patients, control the spread of pathogens (cough etiquette and respiratory hygiene) and minimise time spent in healthcare facilities

2. Provide a package of prevention and care interventions for health workers, including HIV prevention, antiretroviral therapy and isoniazid preventive therapy for HIV-positive health workers

Adapted from [8]. 
TABLE 3 Measures for tuberculosis infection control in healthcare settings: environmental controls

1. Use ventilation systems (natural and mechanical ventilation)

2. Use upper-room ultraviolet germicidal irradiation fixtures with ensured adequate fixture design and air mixing by means of low-velocity ceiling fans

3. Adequate design, construction, renovation, maintenance and use of the health facility are also important

Adapted from [8].

$(\mathrm{ACH})$ by the carbon dioxide decay method. Ambient carbon dioxide levels compared with outdoor levels have been proposed as a way to estimate the fraction of air that has been recently exhaled by other room occupants, another correlate of infection risk if a source case is present [15].

Natural ventilation (e.g. opening windows and using extractors such as roof turbines) has limitations, especially since many buildings have not been specifically designed for this purpose: wind speed and direction are often unpredictable (as are temperature and humidity), and windows often cannot be left open (e.g. for security reasons, vermin or cold weather). Furthermore, the desirable outdoor waiting areas are usually not suitable in cold climates or are difficult to provide in a city environment [15].

Adequately designed mechanical ventilation can overcome some of these difficulties, although it is expensive to install, operate and maintain, and involves technical and maintenance challenges potentially hampering its sustainable use in some countries. A recent comprehensive review on environmental controls by NARDELL [15] discussed the role of air cleaners (that recycle and filter room air) and upper-room germicidal ultraviolet (GUV) in environmental control. Air cleaners, while potentially effective, often underperform (usually adding only a few equivalent $\mathrm{ACH}$ ); in contrast, GUV was recently demonstrated to have an efficacy of $72 \%$ in Peru [29] and $80 \%$ in South Africa [30]. However, GUV fixtures must be properly designed, built, installed and maintained for efficacy and safety, and used together with adequate room air mixing by low-velocity ceiling fans, to be fully effective [15]. New approaches to room GUV dosing, fixture design and output measurement, and the availability of an international maintenance guide [31], make GUV an increasingly attractive option wherever and whenever natural or mechanical ventilation is inadequate [32]. Light-emitting diode GUV is under development that could run on solar or battery power. Among all environmental controls, GUV is the most cost-effective to install and operate. A recent study found that in Vladimir Oblast in the Russian Federation the annual operational cost of one equivalent room air change (removal of $63 \%$ of contaminants) was USD126 for mechanical ventilation, USD143-287 for an air cleaner and as low as USD14 for GUV $[1,14,15]$.

Upper-room GUV with air mixing is relatively inexpensive to install, operate and maintain, and is the most cost-effective environmental control system currently available, especially for retrofitting older buildings without existing ventilation ductwork.

\section{Personal respiratory protection}

The aim of personal respiratory protection is to reduce exposures in personnel working in environments with contaminated air (table 4) [8].

Surgical masks are indicated for patients, while respirators are indicated to protect staff and visitors from inhaling droplet nuclei [13]. Surgical masks worn by patients were shown to reduce TB transmission to guinea pigs by 53\% [33]. They are intended for short-term use and cannot be worn continuously (e.g. while eating or sleeping). Surgical masks stop large particles from becoming infectious droplets at the source.

TABLE 4 Measures for tuberculosis (TB) infection control in healthcare settings: personal respiratory protection

1. Surgical masks prevent infectious particles being expelled by the wearer

2. Particulate respirators protect healthcare workers from inhaling infectious particles

3. When used with administrative and environmental controls, type N95/FFP2 or FFP3 respirators provide additional protection for healthcare workers caring for infectious TB patients

4. Whenever respirators are used, a respirator programme is necessary

Adapted from [8]. 
A tight fit is not required. Surgical masks on patients serve a function similar to cough hygiene using a tissue or hand.

The importance of respirators to protect from inhaling droplet nuclei is highlighted in several guidelines $[9,10,34,35]$ and a recent study [36]. Face-seal leakage is the major limitation of disposable respirators. Effective portable N95/FFP2 standard respirators need to be fit tested: the estimated effectiveness of fit-tested respirators does not exceed $70-80 \%$ due to face-seal leakage [17].

\section{Need for national/subnational and facility planning}

Detailed facility-level plans are necessary to ensure that administrative controls, environmental controls and personal protection are properly implemented at the national and subnational levels [8]. In 2007, BASU et al. [36] attempted to model the extent to which a combination of early diagnosis, effective treatment, isolation of patients and personal protection for staff can prevent TB transmission. Their study investigated the effect of administrative, environmental and personal protection infection control measures on the MDR/ XDR-TB epidemic in the rural community of Tugela Ferry in South Africa using a model incorporating longitudinal inpatient and community-based data collected over 2 years. In the absence of new infection control interventions, approximately $1300 \mathrm{XDR}-\mathrm{TB}$ cases were predicted to occur in the area by the end of 2012, with 50\% due to nosocomial transmission. The use of masks (patients) and respirators (HCWs) alone was predicted to avert $<10 \%$ of cases (as well as preventing transmission among HCWs). A combination of masks and reduced hospitalisation time, with a shift to outpatient treatment, was predicted to prevent up to one-third of XDR-TB cases. Improving ventilation, using rapid drug resistance testing, and ensuring HIV treatment and isolation of TB patients was predicted to prevent $\sim 48 \%$ of XDR-TB cases (range $34-50 \%$ ). For example, the effectiveness of natural ventilation does not apply to the FSU countries because of changes in temperature and humidity. The model depends heavily on the underlying assumptions of intervention efficacy.

A recent evaluation of infection control measures adopted in 88 hospitals in China does not provide evidence on the appropriateness or effectiveness of the measures [37]. Indeed, clinical trial evidence of effectiveness of measures to prevent TB transmission is generally lacking.

\section{Question 1: Which patients are infectious and which factors favour disease transmission?}

The actors: patients, health staff and visitors

The healthcare facility occupants playing a role in TB transmission are the patients, health staff and visitors. Although patients are traditionally seen as index cases transmitting the infection and HCWs as the potential victims to be protected, visitors (and other patients) also need to be considered, both as potential index cases or as individuals who might be infected. Although HCWs are known to be at risk of infection [38], recent reports underline the possibility that they can both infect patients [39] and be infected by them [40]. Interestingly, the report by RicheLDi et al. [40] followed disease transmission in a micro-epidemic originating from a highly infectious sputum smear-positive/culture-positive MDR-TB woman who gave birth in a maternity ward. Of the 92 contacts, 88 only had nosocomial contact, whereas the other four had had both nosocomial and household contact: 41 were neonates, 42 were mothers on the ward and nine were visiting relatives (four husbands and five grandparents).

\section{Who is infectious?}

There is clear evidence that the sources of transmission in hospitals are patients (or health staff) with undetected, untreated TB or patients with known TB, but unknown drug resistance (receiving ineffective therapy). Several studies have documented the prevalence of undetected TB [41, 42]. GeLmanova et al. [43] documented the impact of undiagnosed MDR-TB on the emergence of MDR-TB in Tomsk in Siberia in the Russian Federation (a large territory almost of the same size as Poland). The core problem is that most transmission control efforts focus on patients with known TB, most of who are on effective therapy and therefore no longer infectious, whereas most transmission occurs elsewhere $[14,15]$.

Three historical studies (Bedfordshire in the UK (1948-1952) [44], Rotterdam in the Netherlands (19671969) [45] and Saskatchewan in Canada (1966-1971) [46]) show that untreated sputum smear-positive/ culture-positive individuals are much more infectious to household contacts compared with sputum smear-negative individuals (either sputum culture negative or positive) prior to beginning effective anti-TB treatment. Interestingly, there was not much difference in tuberculin skin test (TST) conversion among the contacts of culture-positive/sputum smear-negative and culture-negative/sputum smear-negative cases, underlining that sputum smear positivity is the better predictor of infectiousness. These studies show that $25-65 \%$ of household contacts exposed to sputum smear-positive/culture-positive cases undergo TST conversion compared with $<10 \%$ of those exposed to sputum smear-negative index cases (either sputum culture negative or positive). A more recent Finnish study found that among 609 contacts of 134 index 
cases (sputum culture positive with positive or negative sputum smear findings), four developed TB within 4 years. All had been in contact with untreated sputum smear-positive index cases [47].

A large Brazilian study of 160 index cases and 934 household contacts reported the rates of TB infection and secondary TB cases among the household contacts of sputum smear-positive/culture-positive cases related to the intensity and location of the exposure before treatment started [48]. The sleeping proximity (same bed, room or house), biological (father or mother) or social (spouse) relationship, and the average number of days per month of contact or hours per day nursing the index case and number of meals shared per day were evaluated as significant variables. A proximity score was developed, consisting of clinical variables correlating with an increased risk of TB infection (presence of cough, pulmonary TB, sputum smear positivity, being the index case's caregiver or mother, sleep location and living in the same house). For intense exposure, TST and interferon- $\gamma$ release assay (IGRA) positivity rates were similar $(81 \%$ versus $77 \%$, respectively, for contacts sleeping in the same bed; $69 \%$ versus $67 \%$, respectively, for contacts sleeping in the same room); for milder exposure, the TST positivity rate was higher than the IGRA positivity rate. The rate of both TST and IGRA positivity increased with the proximity score. Of the 710 individuals who underwent both IGRA and TST, 69\% of contacts sleeping in the same bed were positive for both IGRA and TST compared with $62 \%$ of contacts sleeping in the same room, $54 \%$ of those sleeping in the same house and $39 \%$ of those sleeping in another house $[48,49]$. The rates of secondary TB cases and TB incidence among household contacts increased with the proximity score. The proximity score was also associated with microbiologically confirmed TB disease (sputum smear positive/culture positive). The findings suggest that TB transmission before treatment was due to sputum smear-positive/culture-positive cases, although the study design did not generate information on how many infections or TB cases were specifically due to sputum smear-positive (only) or culture-positive cases.

In a fingerprinting study in California in the USA, $17 \%$ of transmission occurred among contacts of sputum smear-negative index cases before their treatment started [50]. The relative transmission rate from sputum smear-negative versus sputum smear-positive cases was 0.22 (or around one-fifth). The study by ACUNA-VilLaORduna et al. [51] showed that cough aerosol sampling was more predictive of transmission than sputum smear positive alone, presumably because that methodology incorporates the ability to generate aerosol.

Once on effective therapy, sputum smear/culture status no longer predicts transmission. GunNELs et al. [52] reported no difference in infection among household contacts of 86 culture-positive cases (52 were sputum smear negative/culture positive) and 69 culture-negative cases undergoing anti-TB chemotherapy (with 284 and 216 contacts, respectively). The human-to-guinea pig studies of RiLEY $[53,54]$ demonstrated more precisely the rapid effect of treatment on transmission. Smear-positive patients, immediately treated on admission, were $98 \%$ less infectious than smear-positive patients with delayed treatment. More recently, DHARMADHIKARI et al. [55] reported similar responses to standard treatment for MDR-TB.

In summary, transmission is multifactorial, relating to the concentration of viable, infectious bacilli in airborne droplet nuclei, itself the result of source strength, ventilation, proximity, drug susceptibility and, most importantly, the administration of effective treatment. Until effective treatment is provided, sputum smear positivity can be used to estimate infectiousness. We describe later that this is no longer true when the patient starts treatment, as long as the regimen is effective (i.e. there is no undetected resistance when a regimen for susceptible cases is administered) $[14,20]$.

\section{Exposure time needed to generate infection}

Various factors can influence the effectiveness of TB transmission, including factors related to the index case and the contact (discussed later) $[6,8]$. Other important factors include the proximity, frequency and duration of contact. The Rotterdam study demonstrated the importance of proximity in TB transmission: $35 \%$ of close contacts of a sputum smear-positive index case were infected versus $10 \%$ of casual contacts. For sputum smear-negative cases, the proportions were lower ( $10 \%$ and $<10 \%$, respectively) [45]. The core question remains: what are the minimum frequency and duration of contact for infection to occur?

Historical studies in animal models enabled the study of airborne M. tuberculosis in droplet nuclei [53, 54, 56]. In the studies by RiLEY [53, 54], a TB ward with single rooms housing TB untreated patients was connected via a controlled, calibrated closed ventilation circuit to a large exposure chamber containing (exposed) guinea pigs. The animals were monitored by TST and their organs were examined if signs of disease developed. This method allowed the number of infectious doses in the air leading to effective transmission to be quantified. On average, one infectious dose was present in $340 \mathrm{~m}^{3}$ of air. The authors also estimated that the time needed for a nurse to breathe in this volume of air and become infected in the pre-antibiotic era (i.e. have TST conversion) was $12-18$ months, on average. The infectious dose estimate for naturally exposed guinea pigs worked reasonably well for naturally exposed people. 
A cut-off time of $8 \mathrm{~h}$ for exposure to an index case is used in several countries to initiate contact tracing. This approach has no scientific basis, as infection clearly can result from multiple brief exposures (the seconds needed to inhale an infectious particle) [57-60], and the (modest) evidence derives from transmission in airplanes and other closed settings [10, 11, 34, 57, 61, 62].

In airplanes, two major episodes of transmission occurred in a study of 2600 passengers on 191 flights (in nine different aircraft models). The first episode involved the transmission of $M$. tuberculosis from a crew member to colleagues in a flight lasting $>12 \mathrm{~h}[58,63]$. The second episode involved a few passengers seated close to the index case in a flight lasting $>8 \mathrm{~h}[63,64]$. However, the specific conditions in airplanes (i.e. a small air volume, overcrowded, with pre-defined air circulation and limited filtered air exchange while in flight) cannot be extrapolated to other settings.

In the MDR-TB micro-epidemic in a maternity ward in Modena in Italy, RicheLDI et al. [40] reported an average exposure time of $6.05 \mathrm{~h}$ for the 88 main contacts and of $>8 \mathrm{~h}$ for 15 contacts. Of the 88 contacts, 17 became IGRA (ELISPOT) positive. This study was the first to demonstrate the importance of casual nosocomial contacts and a correlation between exposure and TST and IGRA conversion. The sputum smear-positive/culture-positive index case (highly infectious, undiagnosed) remained in the maternity ward (with six beds per room) for 4 days post-natally, alongside other babies and mothers. The mothers slept in the same room and the babies slept separately in a nursery, with no specific infection control measures.

The risk of children becoming TST positive following exposure to sputum smear-positive adults at day care or after-school care facilities was considered low in a Norwegian study: $<3 \%$ of children underwent TST conversion after a median contact period of $6.9 \mathrm{~h}$ [65]. However, a recent Italian study demonstrated that children can become infected within a rather shorter exposure time. A highly contagious paediatrician transmitted $M$. tuberculosis (resulting in several TB cases) in children undergoing vaccination against non-TB diseases, with an exposure time of only 15-20 min [39]. This study confirmed previous findings of rapid transmission in children exposed for $<8 \mathrm{~h}$ while calling for improved workplace safety measures [66].

In a recent household contact study of sputum smear-positive/culture-positive cases performed in Brazil in a setting with a moderate TB incidence, the intensity of exposure and sleep location in relation to the TB index case were directly related to the TST/IGRA response and incidence of secondary TB [48]. The proximity score (a modified version of the Mandalakas score) provided a standardised way to capture the cumulative risk factors of proximity from 10 different variables [48]. These included cough features, existence of pulmonary TB, microbiological status, sleep location and living in the same house.

In summary, the available evidence does not enable the establishment of a cut-off time allowing infection to occur: it is not possible to define a lower time limit to exclude the possibility of $M$. tuberculosis transmission as several factors contribute, including source strength, dilution/ventilation, virulence of the infectious agent and host susceptibility. It is useful to underline that TB infection is a probabilistic and not a deterministic event.

The best approach available to establish infectiousness is the concentric circle or "stone-in-the-pond" approach to contact investigation [61, 67].

\section{Features that influence transmission}

Various factors can influence transmission; these can relate to the index case, the recipient, the bacterial strain or the environment $[6,8]$.

All factors favouring transmission deserve to be adequately evaluated by the clinician, including cough, clinical signs and symptoms, microbiological status (sputum smear, sputum culture, resistance pattern determined by both DST and rapid Xpert MTB/RIF testing), and radiological features.

As the FAST approach maintains, the most dangerous TB cases are the unknown/undiagnosed cases. Therefore, HCWs must keep a high level of clinical suspicion when dealing with patients reporting signs and symptoms compatible with TB (cough lasting for $>2$ weeks, general malaise, night sweats, fever, haemoptysis and weight loss) $[35,68]$. The patient should undergo bacteriological examinations (rapid diagnostic tests, sputum smear, sputum culture and DST to confirm TB and exclude MDR-TB) and chest radiography $[35,69-71]$.

In the case of positive sputum smear findings and Xpert MTB/RIF confirmation of TB, the patient should be rapidly started on an effective TB treatment. If Xpert MTB/RIF testing confirms resistance, the patient should be treated as for MDR-TB. Rapid diagnostic tests for second-line drugs are useful to rapidly diagnose MDR-TB and exclude XDR-TB to avoid empirical treatment with insufficient active drugs $[35,68]$.

The contacts of a TB case should undergo epidemiological evaluation according to the concentric circle/ "stone-in-the-pond" approach [67] and those infected (as evaluated by TST and/or IGRA, in line with 
national policies) should be treated for latent TB infection, as recommended by the WHO, after active TB is ruled out $[35,68,72-74]$.

\section{Question 2: When does an infectious patient become noninfectious after starting treatment?}

The core question for developing a policy on isolation, discharge and management at home for patients is: how long does it take anti-TB chemotherapy to render a patient noninfectious? We will provide evidence showing that effective treatment essentially stops transmission, so that interventions such as isolation, air disinfection and wearing a mask (for patients) or respirator (for health staff and visitors), although important, are less important than sometimes assumed [15].

\section{What is the effect of treatment on infectiousness?}

Rapid diagnosis and effective treatment are the core factors to reduce the infectiousness of TB patients [13, 15 , 17]. Clearly, adequate therapy should be given, in terms of drugs/regimens based on drug susceptibility, administration route, dose, duration, adherence and drug absorption $[35,75,76]$.

Observational studies performed in the pre-MDR era demonstrated that anti-TB chemotherapy can rapidly render patients noninfectious [52-54, 77-81]. Based on this evidence, the 2-week rule after starting treatment was established (as the time needed for adequately treated patients to be considered noninfectious) [77].

Older guidelines tend to focus mainly on the 2-week rule, and still rely on sputum smear and culture conversion results, irrespective of whether effective treatment has been initiated. For example, infection control guidelines of the American Thoracic Society, US Centers for Disease Control and Prevention, and Infectious Diseases Society of America state that patients with pulmonary $\mathrm{TB}$ can be considered noninfectious when: 1) they have received adequate chemotherapy for 2-3 weeks, 2) they show clinical improvement and 3) there is a negligible chance of MDR-TB [9].

This rule was achieved through consensus among experts, who appeared not to consider the important results of Riley's human-to-guinea pig studies (although these were available at the time) [53, 54, 80].

In the first study, Riley et al. [80] observed three to four guinea pig infections per month following exposure to sputum smear-positive, previously treated, drug-resistant TB patients by means of a specially constructed TB ward. When newly diagnosed, previously untreated cases were admitted to the ward, the infections in guinea pigs stopped; they re-started when drug-resistant patients were admitted to the ward. These results were interpreted to indicate that the action of starting adequate treatment for susceptible cases on the day of admission to the ward was highly effective in stopping transmission.

In the second study, RILEY et al. [80] exposed guinea pigs to drug-susceptible and drug-resistant TB patients (MDR-TB did not exist at the time) who had been untreated or treated since diagnosis and admission. The rather weak regimen used at the time (streptomycin, isoniazid and para-aminosalicylic acid) reduced infectiousness of drug-susceptible $M$. tuberculosis by $98 \%$ with an almost immediate effect since treatment was started at the time of admission. However, given the small number of drug-resistant cases, no firm conclusions could be made at that time, but have been made since based on further human-to-guinea pig transmission studies [55].

FENNELLY et al. [56] cultured bacilli from a sample of cough aerosols using a novel method. They found that aerosol cultures from four adequately treated MDR-TB patients declined exponentially, much faster than results from sputum smear microscopy and smear culture. The numbers of patients were small, but the result supports the core role of treatment in reducing infectiousness and the unreliability of sputum smear/culture assays to monitor infectiousness after treatment starts.

EsCOMBE et al. [82] studied guinea pigs exposed to exhaust air from a ward admitting TB/HIV co-infected patients. They showed that transmission occurred from nine patients with unsuspected MDR-TB, inadequately treated because underlying drug resistance was not suspected. A total of 292 guinea pigs were exposed for $>505$ days to exhaust air from 97 TB/HIV co-infected patients with pulmonary TB: 66 were sputum culture positive and 35 were sputum smear positive. Of 125 infected guinea pigs, 122 (98\%) were infected by nine MDR-TB patients who were inadequately treated or whose treatment was delayed. Only three drug-susceptible TB patients infected one guinea pig each: two had delayed treatment and one interrupted treatment due to adverse events. Most TB patients on effective anti-TB treatment did not infect guinea pigs; only patients with undetected MDR-TB infected guinea pigs. This study supports the belief that effective treatment prevents transmission regardless of sputum smear and culture status.

In a recent study, guinea pigs were exposed to exhaust air from rooms hosting presumed MDR-TB cases [55]. In different human-to-guinea pig transmission experiments, the proportion of guinea pigs infected 
ranged from $1 \%$ to $77 \%$. The reasons for the marked inter-experimental variation in transmission rates were unclear: patients had been selected based on similar characteristics associated with transmission (cough, sputum smear positivity and lung cavities) and all were receiving the standard South African MDR-TB treatment regimen. However, the explanation became evident when the fingerprint of several guinea pig isolates matched human isolates of XDR-TB, but not of MDR-TB. In one experiment, a cohort of 27 patients with similar transmission characteristics had infected just one out of 90 guinea pigs subjected to 3 months of continuous exposure. There were no XDR-TB patients in that cohort. As in the aforementioned studies by Riley, in most patients treatment started at the same time that the patient entered the experimental ward (not weeks earlier), again suggesting that effective treatment stops transmission almost immediately, including for MDR-TB. However, the same may not be true for XDR-TB, as effective drugs for MDR-TB such as fluoroquinolones and injectables are ineffective against XDR-TB. An unpublished study showed that supplementing a failed South African MDR regimen with bedaquiline and linezolid (according to the South African protocol) did not reduce transmission to guinea pigs over a 13-day period, but those drugs with pretomanid as part of the NIX regimen (i.e. pretomanid, bedaquiline and linezolid) were effective in rapidly stopping transmission (E. Nardell, Harvard Medical School, Boston, MA, USA; personal communication, 2018) [1, 2].

DhARMADHIKARi et al. [55] and FARMER and RAVIGLIONE [83] concluded that the standard MDR-TB treatment used in South Africa rapidly and effectively suppresses MDR-TB disease transmission, regardless of the sputum smear and culture status, similar to the results of RiLEY et al. [81] and of EsCOMBE et al. [82] for drug-susceptible TB. These studies show the importance of rapidly detecting XDR-TB using rapid genetic tests and the timely initiation of appropriate treatment.

Recent evidence from the Russian Federation demonstrated that rapid diagnosis of MDR-TB by Xpert $\mathrm{MTB} / \mathrm{RIF}$ resulted in a lower incidence of MDR-TB in the population (presumably due to decreased transmission and re-infection in hospitals), and that prolonged infectiousness in a patient under treatment can be due to inadequate chemotherapy, drug resistance and lack of treatment adherence [84-86]. We have been unable to find case reports of transmission from patients under effective treatment $[15,17,87]$.

FitZWATER et al. [88] studied 95 drug-susceptible and -resistant TB cases in Peru treated with standardised short-course chemotherapy under strict directly observed treatment (DOT). The median time to convert sputum culture to a negative result was 38.5 days for the whole cohort. It was not affected by the sputum smear status at baseline. The median time to sputum culture conversion among drug-susceptible TB cases was 37 days; of these, $10 \%$ were still culture positive at day 60 , which predicts the existence of MDR-TB. The results of this study should be interpreted as confirmation that conversion of sputum smear and culture might take time (particularly for MDR-TB cases), but that these outcomes cannot be used to predict transmission once effective treatment has started [15, 17]. Of course, on ineffective treatment, failure to convert sputum and culture (accompanied by deterioration of radiological and clinical indicators) are important clues, so clinical judgement is always required.

Some of the contents of a systematic review of available experimental and epidemiological evidence on the contagiousness of treated patients will be critically discussed later where relevant [89].

\section{Experimental and epidemiological evidence}

Although several studies used sputum smear and sputum culture conversion as indicators of effective treatment [90-92], we underline once more that they cannot be used to evaluate infectiousness [15, 17].

Aerosolisation and drug concentration may play a key role in limiting the spread of disease from patients on effective treatment, as suggested by Loudon and colleagues [21, 22]. In a trial to investigate the mechanism determining the rapid fall in infectiousness of patients after starting treatment, Loudon et al. [22] theorised that evaporation of the droplet nuclei could increase the drug concentration around bacilli. This mechanism might inactivate the bacilli or hamper their capacity to successfully infect human hosts. Although the drug concentration must increase as droplets evaporate, this phenomenon was never confirmed experimentally to reduce the transmission rate.

Only a few studies have evaluated the possibility that index cases under treatment might infect their contacts and only five studies have reported epidemiological evidence [52, 78, 79, 81, 93]. The most well known is the Madras study in India by KамAT et al. [79], which to the best of our knowledge is the only randomised study. The other four studies, performed in the USA, although consistent, could have inadvertently allocated less severe cases to the ambulatory arm (although there is no evidence that biased allocation occurred) $[52,78,81,93]$. The main limitation of all five studies is that $30-65 \%$ of household contacts were already infected at the time treatment started and they did not consider the possibility of re-infection. The proportion of the remaining contacts who remained uninfected ranged from $18 \%$ to $70 \%$ in the different studies. The microbiological status and settings were different, as was treatment duration 
before exposure $[52,78,79,81,93]$. The Madras study found that fewer contacts (both TST positive and negative) of patients treated at home developed TB compared with contacts of patients treated at a sanatorium [79]. Another important finding was that most of the contacts who developed TB did so within the first 3 months (irrespective of whether the index case was treated at home or in the sanatorium). The other four studies had consistent results [52, 78, 81, 93].

In summary, the aforementioned studies do not provide convincing evidence that patients undergoing effective treatment can infect their contacts, regardless of smear or culture status. The studies by GuNNELS et al. [52], Riley [53, 54] and Dharmadhikari et al. [55] clearly demonstrate the rapid effect of adequate treatment on infectiousness.

Which tools do we have to monitor treatment and assess infectiousness?

Sputum smear microscopy and culture are the classic surrogate markers for monitoring treatment and assessing infectiousness [94], and form the most common basis of decisions on the patient's contagiousness before effective treatment starts [95]. Early bactericidal activity is still used as an important measure in controlled clinical trials [94].

In a situation of failure (e.g. when the treatment is not effective, smear and culture do not convert, and clinical and radiological indicators deteriorate) the patient is likely to be still infectious.

Colony numbers (in smear cultures) and time or cycles to positivity in molecular detection can also be used to assess the treatment response in clinical trials, but not for assessing infectiousness unless providing evidence of treatment failure [96].

Radiology (number and extension of pulmonary cavities) can also contribute to treatment success monitoring.

Although pharmacokinetics/pharmacodynamics and therapeutic drug monitoring are not considered treatment monitoring tools, they represent additional resources to ensure adequate dosing, thus aiding effective treatment. They have been recently recommended, for the first time, in the North American TB guidelines [75, 97]. Genetic tests, which are core tools for diagnosis, cannot be used to monitor infectiousness due the high rate of false-positive results (caused by the genetic material of dead bacilli) [98-100].

In acknowledging the limitations of sputum smear as an initial diagnostic tool and to guide treatment decisions, BEHR et al. [50] suggested that interpretations of infectiousness need to be guided by clinical suspicion and the HIV serostatus of contacts. Their study also revealed that treatment was prescribed 12 days later to sputum smear-negative patients than to those positive.

FORTÚN et al. [101] measured the time to sputum smear and culture conversion (34 and 38 days after the start of treatment, respectively), and found that $53 \%$ of patients underwent culture conversion within the first 4 weeks of treatment. Thus, the time to sputum smear and culture conversion is useful to evaluate treatment effectiveness, but not the patient's infectiousness. HernándEZ-Garduño et al. [102] identified high colony counts, lung cavities and a prolonged symptomatic period as factors associated with persistent culture positivity in treated cases, in addition to drug resistance.

In summary, monitoring infectiousness is simply ensuring that treatment, based on DST, appears to be effective based on clinical response and, eventually, by follow-up culture.

Question 3: What can render the patient noninfectious more or less rapidly?

Several factors influence the patient's response to treatment positively or negatively and these need to be coupled to the infection control measures.

Bacterial load/disease site

Patients with extensive cavitary lung disease and high bacterial load are highly infectious and remain infectious for longer.

\section{Nutrition/immunity status}

The roles of nutrition and immunity in combating TB are well known since the sanatoria era. Interventions to improve nutrition and to restore immunity when necessary (i.e. antiretroviral treatment for TB/HIV co-infected patients) are likely to help [103]. However, there is no evidence to support their role in transmission. 
People-centred approach

Enhancing treatment adherence (DOT/video-observed treatment) can improve the patient's response to treatment $[35,104]$. Although plausible (adherence is likely to reduce infectiousness, given the core role of effective treatment), no evidence is currently available to support a relationship between a people-centred approach and the treatment response.

\section{Immunotherapy/host-directed therapies}

New approaches in this area have been proposed, but there is insufficient evidence on their clinical utility [103].

\section{Bactericidal potentialities of treatment regimens}

The bactericidal capacity of the anti-TB regimen in use is likely to reduce the infectiousness period, although there is no experimental evidence to support this. Bactericidal drugs can reduce the number of colonies, but this may not be the only mechanism involved in reducing infectiousness (e.g. the bacilli growing in culture may be not infectious) $[76,105]$.

\section{Improved treatment programmes}

All efforts to improve the quality of treatment (e.g. high-quality drugs, correct doses and regimens, and use of therapeutic drug monitoring) are likely to be useful in reducing infectiousness, although there is no formal evidence to support this.

\section{Implementation of WHO guidance}

Sustainable implementation of administrative, environmental and personal protection measures to reduce the duration and degree of infectiousness are prerequisites for health units admitting MDR-TB patients (see later for details) $[8,36,104,106]$.

\section{Active screening}

The administrative approach of active screening for undetected TB and drug resistance followed by prompt effective therapy is most likely to result in sustainable reductions of transmission.

\section{Question 4: Which patients need hospital admission (and respiratory isolation) because of their infectiousness?}

There is evidence that even patients who remain infectious can be managed at home if treatment is certain to be effective [104]. More evidence is becoming available on the effectiveness (and cost-effectiveness [107]) of decentralised and ambulatory-based programmes to manage MDR/XDR-TB [108-113]. Through the support of visiting nurses, patient-centred programmes have improved case detection rates, continuity of care and treatment outcomes [114]. This section will revise the available evidence to clarify which patients need hospital admission [115].

The Madras study and others demonstrated no differences in the proportion of TST-negative contacts who developed TB following the index case's hospital admission versus home care [52, 79, 116, 117]. Although the treatment regimen was very different from those used today and the setting at high incidence of TB, this study had a profound impact on subsequent policy decisions [79].

In the Madras study, the effect of isoniazid and para-aminosalicylic acid was compared in patients treated under good conditions in a sanatorium (good quality accommodation, nursing care, a nutritious diet and prolonged bed rest) versus those treated under poorer conditions at home [79]. Treatment results were similar in terms of radiological/microbiological outcomes and the proportion of relapses after 4 years. The rate of bacteriological conversion was also similar ( $90 \%$ sputum smear and culture conversion within 4 months): at 2 months, $45 \%$ of home-based patients underwent sputum smear and culture conversion versus $49 \%$ of sanatorium-based patients, while at 4 months the proportions were $89 \%$ and $93 \%$, respectively. Close family contacts were followed-up for 5 years: there was no difference in TB incidence between hospital and family contacts. The TB rate among contacts within 5 years was: TST positivity, nine out of $86(10.5 \%)$ home contacts versus 10 out of $87(11.5 \%)$ sanatorium contacts; TST negativity, 15 out of 159 (9.4\%) home contacts versus 28 out of 177 (15.8\%) sanatorium contacts. Notably, at the time MDR-TB was not yet widespread, around half of the contacts were already TST positive at the time of index case diagnosis and the bactericidal capacity of the treatment regimen was low compared with modern regimens.

BRooks et al. [78] reported no conversions among household contacts following the home management of 21 patients discharged after 23 days of initial hospital treatment; 19 out of 21 were still sputum smear positive when discharged. Menzies and colleagues recommended that patients should at least show initial signs of clinical improvement (clinical, radiological and microbiological improvements, with reduced 
bacilli count in sputum smear microscopy) before being discharged for outpatient management [89, 118]. Experiences in the Russian Federation [43] and other countries (including South Africa) [36, 108-111, $113,119]$ show the dangers of in-hospital transmission and the advantages of home care. Home care can be initiated at the start of treatment if the main condition that effective treatment has been promptly initiated within community-based TB or MDR-TB programmes has been satisfied. BAssiLi et al. [120] evaluated the effectiveness of MDR-TB management in 35 studies (25 in hospital and eight at home) and 14478 patients treated between 1973 and 2007. The treatment success rate was $65.5 \%$ and $66.7 \%$ for hospital and home care, respectively, with no differences for any treatment outcomes related to the care model. Others found no difference in sputum smear conversion rates and treatment outcomes for ambulatory-managed versus hospital-managed patients in Macedonia [121].

Boyd et al. [95] investigated the time to treatment for rifampicin-resistant cases, selecting 53 studies, 83 cohorts and 13034 patients: $76 \%$ of them started treatment with second-line anti-TB drugs. The weighted mean time from sputum collection to treatment was 81 days, being shorter for patients managed in ambulatory than hospital settings (57 versus 86 days). The treatment delay was much shorter when rapid genetic methods were used compared with phenotypic DST (38 versus 108 days). The FAST approach can, then, reduce the delay between detection and the initiation of effective treatment through active case finding, making an important contribution to reducing transmission.

In a qualitative study, HORTER et al. [122] showed that both MDR-TB patients and health staff preferred home-based to hospital-based care. Padayatchi and Friedland [123] underlined that simple, cheap strategies (e.g. opening windows, sitting in the open, covering the mouth when coughing and using respirators) might reduce transmission. However, these interventions are less effective than the prompt initiation of effective treatment $[15,17,124]$.

LOVEDAY et al. [110] provided evidence on the advantage of treating TB at home, even in settings where HIV infection is highly prevalent. The results of a prospective study comparing community-based versus centralised case management for MDR-TB patients demonstrated higher success rates with the community approach.

VAN CUTSEM et al. [124] emphasised the importance of early diagnosis and treatment to reduce infectiousness, and of decentralised models of care to reduce transmission. They described barriers to adherence to infection control principles, including interpersonal factors and those related to the health facility and health system. Yuen et al. [125] used similar arguments supporting active case finding and prompt treatment to reduce transmission.

JENKINs et al. [126] demonstrated that MDR-TB can appear rapidly during treatment in Moldova: between $7.2 \%$ and $9.2 \%$ of $\mathrm{TB}$ cases (initially non-MDR-TB) acquired this status during treatment (half within 3 months of starting treatment). At least $80 \%$ of them were hospitalised. Two main reasons for the increasing MDR-TB epidemic were identified: re-infection (from undetected sources) and the delay in adequately treating patients after DST (i.e. a delay in DST delays the initiation of effective MDR-TB treatment). In confirming the results of the Tomsk study [43] and of another Russian experience (Grigory Volchenkov, Vladimir Oblast TB Dispensary, Vladimir, Russian Federation; personal communication, 2014) the authors concluded that ambulatory care and universal access to rapid diagnostic testing would reduce nosocomial transmission.

\section{TABLE 5 Criteria for hospital admission}

\section{Main criteria [2, 104]}

- Complications of TB le.g. respiratory failure and conditions requiring surgical interventions, such as haemorrhage, pneumothorax and pleural effusion)

- Severe forms of TB (i.e. TB meningitis) and/or severe clinical manifestations of comorbidities (e.g. liver disease, renal disease and uncontrolled diabetes)

- Life-threatening and serious medical events resulting from adverse reactions to anti-TB drugs (e.g. life-threatening arrhythmias, psychosis, renal failure and hearing loss)

Additional criteria [2, 127, 128]

- Patients for whom effective and safe anti-TB treatment cannot be ensured in an outpatient, community or home setting (i.e. homelessness, overcrowding, exposure of children aged $<5$ years and pregnant women in the household)

- When there are accessibility problems (i.e. patient lives far from an outpatient facility)

- Where there is nonadherence to treatment Ithis can be considered in some settings as a last resort once all other care options have been used/applied exhaustively, based on the legal framework in force)

TB: tuberculosis. 
TABLE 6 Features of a hospital admitting tuberculosis (TB) and multidrug-resistant (MDR)/ extensively drug-resistant (XDR)-TB patients

- Clinical expertise in TB and MDR/XDR-TB management (including for directly observed treatment)

- Laboratory results from laboratories with a robust external quality assurance system in place

- Respiratory isolation capacity and adequate infection control measures (including the recommended 12 air changes per hour

- Personal protection measures (i.e. respirators) available within well-designed personal protection programmes, including staff awareness/education and respirator fit testing

- Open spaces that allow patients to socialise without the risk of Mycobacterium tuberculosis transmission [130]

- An adequate number of staff trained and supervised to adhere to administrative and personal protection measures of infection control

- A patient-centred approach (psychological support, palliative care, link with home care and social services for the post-discharge home care phasel

A recent WHO document emphasised the lack of significant differences in treatment outcomes between inpatient and outpatient models of care [104]. Unless there is a clinical or public health need, people with presumably infectious TB or confirmed pulmonary TB should not be admitted to hospital for diagnostic tests or care.

Theoretically, in low TB/MDR-TB incidence countries, individual patients prior to the initiation of effective treatment may be accommodated in single negative-pressure ventilation rooms; however, this is impossible where TB incidence is high (e.g. in the FSU countries). Therefore, hospital admission should be reserved for those TB or MDR-TB cases really needing it.

\section{Criteria for hospital admission}

A proportion of cases still need to be admitted for medical reasons, including severe cases, life-threatening conditions, comorbidities, psychiatric problems and adverse drug reactions, and for social or logistical reasons (table 5) [129].

\section{Features of a hospital admitting TB and MDR/XDR-TB patients}

A patient admitted to hospital should never represent a source of transmission to other patients, health staff and visitors. Therefore, the hospital needs to offer adequate infection control measures in addition to core services (table 6) $[1,2]$.

The WHO suggests that people deemed to be at a low risk of rifampicin-resistant TB and MDR-TB should be placed in single rooms, and that those at a high risk should ideally be accommodated in a negative-pressure room while rapid diagnostic tests are urgently performed until effective treatment starts [104].

\section{Question 5: What are the research needs?}

As more than half of MDR-TB cases result from transmission and the remainder from the selection of drug-resistant mutants, the two core research priorities are to reduce transmission and prevent drug resistance. Both are included in the FAST approach $[13,15,131]$.

More information is needed on how drugs stop transmission, apart from their bactericidal effects. It is also important to discover effective (and cost-effective) screening approaches for undetected TB (e.g. active screening through cough surveillance, digital radiology and breathe tests that do not require sputum). Host-directed therapy is also an emerging area of research, e.g. evaluating the role of statin or metformin-like drugs in preventing or limiting TB in the household contacts of highly drug-resistant patients.

Finally, MDR-TB treatment will inevitably generate XDR-TB cases among treatment failures; unfortunately, some of these patients will be incurable and need palliative care. More research is needed to identify models to ensure that untreatable XDR-TB patients receive the necessary level of comfort (while preventing transmission) at home or in palliative care institutions [106].

\section{Conclusions}

This review indicates how important is to implement the principle of the FAST approach beyond implementing administrative controls, environmental controls and personal protection in order to limit transmission, and suggests some relevant policy elements recently adopted by the WHO Regional Office for Europe (table 7). 
TABLE 7 Main policy elements derived from the present review

- Infection control planning is needed at the national/subnational and facility levels.

- Three groups of actors should be considered: patients, health staff and visitors.

- The cut-off value of $8 \mathrm{~h}$ exposure is not useful to activate contact tracing. Criteria related to the intensity, frequency and duration of exposure should guide the contact-tracing plan following exposure to an infectious TB patient based on the concentric circle/"stone-in-the-pond" approach.

- Sputum smear microscopy is a quick, cheap tool for assessing pre-treatment infectiousness, although it has limitations. Sputum culture is useful to demonstrate the viability of bacilli, although results take 2-3 weeks. Neither sputum nor culture can be used to predict infectiousness once the treatment starts.

- Although sputum smear and culture tests can still be positive $>2$ weeks after treatment starts, the available evidence shows that infectiousness drops very rapidly if adequate treatment is implemented; however, this is difficult to quantify.

- It is easier to provide adequate treatment when the Mycobacterium tuberculosis strain is drug susceptible. The real risk is infection from undetected MDR-TB or XDR-TB cases.

- New rapid molecular diagnostic techniques (i.e. Xpert MTB/RIF and line probe assays) should be systematically used to promptly identify drug-resistant patients so that adequate treatment can be rapidly started, thus reducing the period of infectiousness.

- Stringent isolation criteria are necessary for presumed and confirmed XDR-TB patients for whom treatment does not rapidly prevent transmission. Existing community-based programmes have shown that MDR-TB patients can also be managed at home because effective treatment rapidly reduces their infectiousness.

- Although outpatient management is recommended in principle, a proportion of TB patients (e.g. clinically severe cases) will need hospital admission. Any hospital admitting TB patients should offer adequate infection control and quality patient-centred management.

- FAST (Find cases Actively by cough surveillance and rapid molecular sputum testing, Separate safely, and Treat effectively based on rapid drug susceptibility testing) is the strategy recently proposed to minimise the risk that undetected TB cases further transmit $M$. tuberculosis.

TB: tuberculosis; MDR: multidrug-resistant; XDR: extensively drug-resistant. Adapted from [2].

Active case finding, rapid diagnosis (including drug resistance) and prompt effective therapy are now recognised as the most important interventions to stop transmission. Most infection control attention is on diagnosed TB patients, ideally on effective treatment, whereas most transmission is from patients with unsuspected TB or unsuspected drug resistance not on effective treatment.

Acknowledgements: This article belongs to the scientific activities of the WHO Collaborating Centre for Tuberculosis and Lung Diseases, Tradate, ITA-80, 2017-2020-GBM/RC/LDA and of the Global Tuberculosis Network (GTN), Infection Control Committee (Chair: Edward Nardell).

Conflict of interest: None declared.

\section{References}

1 Nardell E, Volchenkov G. Transmission control: a refocused approach. In: Migliori GB, Bothamley G, Duarte R, et al., eds. Tuberculosis (ERS Monograph). Sheffield, European Respiratory Society, 2018; pp. 364-380.

2 Migliori GB, D'Ambrosio L, Centis R, et al. Guiding Principles to Reduce Tuberculosis Transmission in the WHO European Region. Copenhagen, WHO Regional Office for Europe, 2018.

3 World Health Organization Regional Office for Europe. Roadmap to Prevent and Combat Drug-resistant Tuberculosis. The Consolidated Action Plan to Prevent and Combat Multidrug and Extensively Drug-resistant Tuberculosis in the WHO European Region 2011-2015. Copenhagen, WHO Regional Office for Europe, 2011.

4 European Centre for Disease Prevention and Control, World Health Organization Regional Office for Europe. Tuberculosis Surveillance and Monitoring in Europe 2018-2016 Data. Stockholm, ECDC, 2018.

5 Estebesova A. Systematic Review of Infection Prevention and Control Policies and Nosocomial Transmission of Drug-resistant Tuberculosis. 2013. http://scholarworks.gsu.edu/iph_theses/312 Date last accessed: February 20, 2019.

6 Rieder HL. Epidemiologic Basis of Tuberculosis Control. Paris, International Union Against Tuberculosis and Lung Disease, 1999.

7 Frieden T, ed. Toman's Tuberculosis: Case Detection, Treatment, and Monitoring. Questions and Answers, 2nd Edn. Geneva, WHO, 2004.

8 World Health Organization. Policy on TB Infection Control in Health-care Facilities, Congregate Settings and Households. Geneva, WHO, 2009.

9 Jensen PA, Lambert LA, Iademarco MF, et al. Guidelines for preventing the transmission of Mycobacterium tuberculosis in health-care settings, 2005. MMWR Recomm Rep 2005; 54 (RR-17): 1-141.

10 National Institute for Health and Care Excellence. Tuberculosis: Prevention, Diagnosis, Management and Service Organisation. TB Clinical Guideline Update. 2016. www.nice.org.uk/guidance/ng33/evidence/full-guideline-pdf80851860868 Date last accessed: February 20, 2019.

11 East Cheshire NHS Trust. Tuberculosis: Infection Prevention and Control Policy, Version 5. 2018. www. eastcheshire.nhs.uk/About-The-Trust/policies/I/IC\%20Tuberculosis\%20Prevention\%20and\%20Control\%20ECT00 2970.pdf Date last accessed: February 20, 2019. 
12 European Centre for Disease Prevention and Control. Management of Contacts of MDR TB and XDR TB Patients. Stockholm, ECDC, 2012.

13 Yates TA, Khan PA, Knight GM, et al. The transmission of Mycobacterium tuberculosis in high burden settings. Lancet Infect Dis 2016; 16: 227-238.

14 Barrera E, Livchits V, Nardell E. F-A-S-T: a refocused, intensified, administrative tuberculosis transmission control strategy. Int J Tuberc Lung Dis 2015; 19: 381-384.

15 Nardell EA. Indoor environmental control of tuberculosis and other airborne infections. Indoor Air 2016; 26: 79-87.

16 Nathavitharana RR, Daru P, Barrera AE, et al. FAST implementation in Bangladesh: high frequency of unsuspected tuberculosis justifies challenges of scale-up. Int J Tuberc Lung Dis 2017; 21: 1020-1025.

17 Dheda K, Gumbo T, Maartens G, et al. The epidemiology, pathogenesis, transmission, diagnosis, and management of multidrug-resistant, extensively drug-resistant, and incurable tuberculosis. Lancet Respir Med 2017; 5: 291-360.

18 Houk VH, Kent DC, Baker JH, et al. The Byrd study. In-depth analysis of a micro-outbreak of tuberculosis in a closed environment. Arch Environ Health 1968; 16: 4-6.

19 Houk VN, Baker JH, Sorensen K, et al. The epidemiology of tuberculosis infection in a closed environment. Arch Environ Health 1968; 16: 26-35.

20 Nardell EA. Air sampling for tuberculosis: homage to the lowly guinea pig. Chest 1999; 116: 1143-1145.

21 Loudon RG, Spohn SK. Cough frequency and infectivity in patients with pulmonary tuberculosis. Am Rev Respir Dis 1969; 99: 109-111.

22 Loudon RG, Bumgarner LR, Lacy J, et al. Aerial transmission of mycobacteria. Am Rev Respir Dis 1969; 100: $165-171$.

23 Lever MS, Williams A, Bennett AM. Survival of mycobacterial species in aerosols generated from artificial saliva. Lett Appl Microbiol 2000; 31: 238-241.

24 Xie X, Li Y, Chwang AT, et al. How far droplets can move in indoor environments - revisiting the Wells evaporation-falling curve. Indoor Air 2007; 17: 211-225

25 Menzies D, Fanning A, Yuan L, et al. Hospital ventilation and risk for tuberculous infection in Canadian health care workers. Canadian Collaborative Group in Nosocomial Transmission of TB. Ann Intern Med 2000; 133: 779-789.

26 Escombe AR, Oeser CC, Gilman RH, et al. Natural ventilation for the prevention of airborne contagion. PLoS Med 2007; 4: e68.

27 Cox H, Escombe R, McDermid C, et al. Wind-driven roof turbines: a novel way to improve ventilation for TB infection control in health facilities. PLoS One 2012; 7: e29589.

28 Shenoi SV, Escombe AR, Friedland G. Transmission of drug-susceptible and drug-resistant tuberculosis and the critical importance of airborne infection control in the era of HIV infection and highly active antiretroviral therapy rollouts. Clin Infect Dis 2010; 50: Suppl. 3, S231-S237.

29 Escombe AR, Moore DA, Gilman RH, et al. Upper-room ultraviolet light and negative air ionization to prevent tuberculosis transmission. PLoS Med 2009; 6: e43.

30 Mphaphlele M, Dharmadhikari AS, Jensen PA, et al. Institutional tuberculosis transmission. controlled trial of upper room ultraviolet air disinfection: a basis for new dosing guidelines. Am J Respir Crit Care Med 2015; 192: 477-484.

31 Vincent RL. End TB Transmission Initiative: Maintenance of Upper-room Germicidal Ultraviolet (GUV) Air Disinfection Systems for TB Transmission Control. 2017. www.stoptb.org/wg/ett/assets/documents/ MaintenanceManual.pdf Date last accessed: March 30, 2019.

32 Linnes JC, Rudnick SN, Hunt GM, et al. Eggcrate UV: a whole ceiling upper-room ultraviolet germicidal irradiation system for air disinfection in occupied rooms. Indoor Air 2014; 24: 116-124.

33 Dharmadhikari AS, Mphahlele M, Stoltz A, et al. Surgical face masks worn by patients with multidrug-resistant tuberculosis: impact on infectivity of air on a hospital ward. Am J Respir Crit Care Med 2012; 185: 1104-1109.

34 Communicable Diseases Network Australia. CDNA National Guidelines for Public Health Units - Management of TB. 2015. www.health.gov.au/internet/main/publishing.nsf/Content/D140EDF48C0A0CEACA257BF0001A3537/\$File/ TB-2.0-april2015.pdf Date last accessed: February 20, 2019.

35 Migliori GB, Sotgiu G, Rosales-Klintz S, et al. ERS/ECDC Statement: European Union Standards for Tuberculosis Care, 2017 update. Eur Respir J 2018; 51: 1702678

36 Basu S, Andrews JR, Poolman EM, et al. Prevention of nosocomial transmission of extensively drug-resistant tuberculosis in rural South African district hospitals: an epidemiological modelling study. Lancet 2007; 370: 1500-1507.

37 Chen B, Liu M, Gu H, et al. Implementation of tuberculosis infection control measures in designated hospitals in Zhejiang Province, China: are we doing enough to prevent nosocomial tuberculosis infections? BMJ Open 2016; 6: e010242.

38 Sotgiu G, Sorete Arbore A, Cojocariu V, et al. High risk of tuberculosis in health care workers in Romania. Int J Tuberc Lung Dis 2008; 12: 606-611.

39 Luzzati R, Migliori GB, Zignol M, et al. Children under 5 years are at risk for tuberculosis after an occasional contact with highly contagious patients: an out-patient outbreak from a smear positive health care worker. Eur Respir J 2017; 50: 1701414.

40 Richeldi L, Ewer K, Losi M, et al. T cell-based tracking of multidrug resistant tuberculosis infection after brief exposure. Am J Respir Crit Care Med 2004; 170: 288-295.

41 Willingham FF, Schmitz TL, Contreras M, et al. Hospital control and multidrug-resistant pulmonary tuberculosis in female patients, Lima, Peru. Emerging Infect Dis 2001; 7: 123-127.

42 Bates M, O'Grady J, Mwaba P, et al. Evaluation of the burden of unsuspected pulmonary tuberculosis and co-morbidity with noncommunicable diseases in sputum producing adult inpatients. PLoS One 2012; 7: e40774.

43 Gelmanova IY, Keshavjee S, Golubchikova VT, et al. Barriers to successful tuberculosis treatment in Tomsk, Russian Federation: non-adherence, default and the acquisition of multidrug resistance. Bull World Health Organ 2007; 85: 703-711.

44 Shaw JB, Wynn-Williams N. Infectivity of pulmonary tuberculosis in relation to sputum status. Am Rev Tuberc 1954; 69: 724-732. 
van Geuns HA, Meijer J, Styblo K. Results of contact examination in Rotterdam, 1967-1969. Bull Int Union Tuberc 1975; 50: 107-121.

Grzybowski S, Barnett GD, Styblo K. Contacts of cases of active pulmonary tuberculosis. Bull Int Union Tuberc 1975; 50: 90-106.

Liippo KK, Kulmala K, Tala EO. Focusing tuberculosis contact tracing by smear grading of index cases. Am Rev Respir Dis 1993; 148: 235-236.

Acuña-Villaorduña C, Jones-López EC, Fregona G, et al. Intensity of exposure to pulmonary tuberculosis determines risk of tuberculosis infection and disease. Eur Respir J 2018; 51: 1701578.

Barcellini L, Borroni E, Brown J, et al. First evaluation of QuantiFERON-TB Gold Plus performance in contact screening. Eur Respir J 2016; 48: 1411-1419.

Behr MA, Warren SA, Salamon H, et al. Transmission of Mycobacterium tuberculosis from patients smear-negative for acid-fast bacilli. Lancet 1999; 353: 444-449.

Acuna-Villaorduna CJ, Schmidt-Castellani LG, White L, et al. Cough aerosol cultures of Mycobacterium tuberculosis predict quantitative IGRA readouts in household contacts of pulmonary TB patients in Brazil. Am $J$ Respir Crit Care Med 2017; 195: A7629.

Gunnels JJ, Bates JH, Swindoll H. Infectivity of sputum-positive tuberculous patients on chemotherapy. Am Rev Respir Dis 1974; 109: 323-330.

Riley RL. The hazard is relative. Am Rev Respir Dis 1967; 96: 623-625.

Riley RL. The contagiosity of tuberculosis. Schweiz Med Wochenschr 1983; 113: 75-79.

Dharmadhikari AS, Mphahlele M, Venter K, et al. Rapid impact of effective treatment on transmission of multidrug-resistant tuberculosis. Int J Tuberc Lung Dis 2014; 18: 1019-1025.

Fennelly KP, Martyny JW, Fulton KE, et al. Cough-generated aerosols of Mycobacterium tuberculosis: a new method to study infectiousness. Am J Respir Crit Care Med 2004; 169: 604-609.

National Tuberculosis Controllers Association, Centers for Disease Control and Prevention. Guidelines for the investigation of contacts of persons with infectious tuberculosis. Recommendations from the National Tuberculosis Controllers Association and CDC. MMWR Recomm Rep 2005; 54 (RR-15): 1-37.

Driver CR, Valway SE, Morgan WM, et al. Transmission of Mycobacterium tuberculosis associated with air travel. JAMA 1994; 272: 1031-1035.

Gerald LB, Tang S, Bruce F, et al. A decision tree for tuberculosis contact investigation. Am J Respir Crit Care Med 2002; 166: 1122-1127.

Kenyon TA, Valway SE, Ihle WW, et al. Transmission of multidrug-resistant Mycobacterium tuberculosis during a long airplane flight. N Engl J Med 1996; 334: 933-938.

Erkens CG, Kamphorst M, Abubakar I, et al. Tuberculosis contact investigation in low prevalence countries: a European consensus. Eur Respir J 2010; 36: 925-949.

World Health Organization. Tuberculosis and Air Travel: Guidelines for Prevention and Control. 2nd Edn. Geneva, WHO, 2006.

Kotila SM, Payne Hallström L, Jansen N, et al. Systematic review on tuberculosis transmission on aircraft and update of the European Centre for Disease Prevention and Control risk assessment guidelines for tuberculosis transmitted on aircraft (RAGIDA-TB). Euro Surveill 2016; 21: 30114.

McFarland JW, Hickman C, Osterholm M, et al. Exposure to Mycobacterium tuberculosis during air travel. Lancet 1993; 342: 112-113.

Døllner H, Ramm CT, Harstad I, et al. Risk of developing tuberculosis after brief exposure in Norwegian children: results of a contact investigation. BMJ Open 2012; 2: e001816.

Valway SE, Sanchez MPC, Shinnick TF, et al. An outbreak involving extensive transmission of a virulent strain of Mycobacterium tuberculosis. N Engl J Med 1998; 338: 633-639.

Veen J. Microepidemics of tuberculosis: the stone-in-the-pond principle. Tubercle Lung Dis 1992; 73: 73-76.

Wealth Organization. Compendium of WHO Guidelines and Associated Standards: Ensuring Optimum Delivery of the Cascade of Care for Patients with Tuberculosis. Geneva, WHO, 2017.

24: 73-85. diagnostics and biomarkers. Lancet Infect Dis 2018; 18: e199-e210.

WHO Regional Office for Europe. Algorithm for Laboratory Diagnosis and Treatment-monitoring of Pulmonary Tuberculosis and Drug-resistant Tuberculosis using State-of-the-art Rapid Molecular Diagnostic Technologies. Copenhagen, WHO Regional Office for Europe, 2017.

Gilpin C, Korobitsyn A, Migliori GB, et al. The World Health Organization standards for tuberculosis care and management. Eur Respir J 2018; 51: 1800098.

Getahun H, Matteelli A, Abubakar I, et al. Management of latent Mycobacterium tuberculosis infection: WHO guidelines for low tuberculosis burden countries. Eur Respir J 2015; 46: 1563-1576.

World Health Organization. Latent Tuberculosis Infection: Updated and Consolidated Guidelines for Programmatic Management. Geneva, WHO, 2018.

Nahid P, Dorman SE, Alipanah N, et al. Official American Thoracic Society/Centers for Disease Control and Prevention/Infectious Diseases Society of America clinical practice guidelines: treatment of drug-susceptible tuberculosis. Clin Infect Dis 2016; 63: e147-e195.

Caminero JA, Sotgiu G, Zumla A, et al. Best drug treatment for multidrug resistant and extensively drug-resistant tuberculosis. Lancet Infect Dis 2010; 10: 621-629.

Rouillon A, Perdrizet S, Parrot R. Transmission of tubercle bacilli: the effects of chemotherapy. Tubercle 1976; 57: 275-299.

Brooks SM, Lassiter NL, Young EC. A pilot study concerning the infection risk of sputum positive tuberculosis patients on chemotherapy. Am Rev Respir Dis 1973; 108: 799-804.

Kamat SR, Dawson JJ, Devadatta S, et al. A controlled study of the influence of segregation of tuberculous patients for one year on the attack rate of tuberculosis in a 5-year period in close family contacts in South India. Bull World Health Organ 1966; 34: 517-532. 
Riley RL, Mills CC, Nyka W, et al. Aerial dissemination of pulmonary tuberculosis. A two-year study of contagion in a tuberculosis ward. 1959. Am J Epidemiol 1995; 142: 3-14.

Riley RL, Moodie AS. Infectivity of patients with pulmonary tuberculosis in inner city homes. Am Rev Respir Dis 1974; 110: 810-812.

Escombe AR, Moore DA, Gilman RH, et al. The infectiousness of tuberculosis patients coinfected with HIV. PLoS Med 2008; 5: e188.

Farmer PE, Raviglione MC. Rapid impact of effective chemotherapy on transmission of drug-resistant tuberculosis: pity the guinea pig. Int J Tuberc Lung Dis 2014; 18: 1009-1011.

Goble M, Iseman MD, Madsen LA, et al. Treatment of 171 patients with pulmonary tuberculosis resistant to isoniazid and rifampin. N Engl J Med 1993; 328: 527-532.

Krivinka R, Drápela J, Kubík A, et al. Epidemiological and clinical study of tuberculosis in the district of Kolín Czechoslovakia. Second report (1965-1972). Bull World Health Organ 1974; 51: 59-69.

Styblo K, Daňková D, Drápela J, et al. Epidemiological and clinical study of tuberculosis in the district of Kolín, Czechoslovakia. Bull World Health Organ 1967; 37: 819-874.

Miller AC, Livchits V, Ahmad Khan F, et al. Turning off the tap: using the FAST approach to stop the spread of drug-resistant tuberculosis in the Russian Federation. J Infect Dis 2018; 218: 654-658.

Fitzwater SP, Caviedes L, Gilman RH, et al. Prolonged infectiousness of tuberculosis patients in a directly observed therapy short-course program with standardized therapy. Clin Infect Dis 2010; 51: 371-378.

Menzies D. Effect of treatment on contagiousness of patients with active pulmonary tuberculosis. Infect Control Hosp Epidemiol 1997; 18: 582-586.

Singapore Tuberculosis Service, British Medical Research Council. Controlled trial of intermittent regimens of rifampicin plus isoniazid for pulmonary tuberculosis in Singapore. Lancet 1975; 2: 1105-1109.

Tuberculosis Research Centre. Study of chemotherapy regimens of 5 and 7 months' duration and the role of corticosteroids in the treatment of sputum-positive patients with pulmonary tuberculosis in South India. Tubercle 1983; 64: 73-91.

Kim TC, Blackman RS, Heatwole KM, et al. Acid-fast bacilli in sputum smears of patients with pulmonary tuberculosis. Prevalence and significance of negative smears pretreatment and positive smears post-treatment. Am Rev Respir Dis 1984; 129: 264-268.

Abeles H. Early hospital discharge of tuberculous patients with sputum containing acid-fast bacilli on microscopic examination. Am Rev Respir Dis 1973; 108: 975-977.

Diacon AH, Dawson R, von Groote-Bidlingmaier F, et al. 14-day bactericidal activity of PA-824, bedaquiline, pyrazinamide, and moxifloxacin combinations: a randomized trial. Lancet 2012; 380: 986-993.

Boyd R, Ford N, Padgen P, et al. Time to treatment for rifampicin-resistant tuberculosis: systematic review and meta-analysis. Int J Tuberc Lung Dis 2017; 21: 1173-1180.

Tiberi S, De Lorenzo S, Centis R, et al. Bedaquiline in MDR/XDR-TB cases: first experience on compassionate use. Eur Respir J 2014; 43: 289-292.

Srivastava S, Peloquin CA, Sotgiu G, et al. Therapeutic drug management: is it the future of multidrug-resistant tuberculosis treatment? Eur Respir J 2013; 42: 1449-1453.

Ignatyeva $\mathrm{O}$, Balabanova $\mathrm{Y}$, Nikolayevskyy $\mathrm{V}$, et al. Resistance profile and risk factors of drug resistant tuberculosis in the Baltic countries. Tuberculosis 2015; 95: 581-588.

Miotto P, Tessema B, Tagliani E, et al. A standardised method for interpreting the association between mutations and phenotypic drug resistance in Mycobacterium tuberculosis. Eur Respir J 2017; 50: 1701354.

Theron G, Venter R, Calligaro G, et al. Xpert MTB/RIF results in patients with previous tuberculosis: can we distinguish true from false positive results? Clin Infect Dis 2016; 62: 995-1001.

Fortún J, Martín-Dávila P, Molina A, et al. Sputum conversion among patients with pulmonary tuberculosis: are there implications for removal of respiratory isolation? J Antimicrob Chemother 2007; 59: 794-798.

Hernández-Garduño E, Cook V, Kunimoto D, et al. Transmission of tuberculosis from smear negative patients: a molecular epidemiology study. Thorax 2004; 59: 286-290.

Tiberi S, du Plessis N, Walzl G, et al. Tuberculosis: progress and advances in development of new drugs, treatment regimens and host-directed therapies. Lancet Infect Dis 2018; 18: e183-e198.

WHO Regional Office for Europe. A People-centred Model of Tuberculosis Care. A Blueprint for Eastern European and Central Asian Countries. Copenhagen, WHO Regional Office for Europe, 2017.

extensively drug-resistant tuberculosis cases. Eur Respir J 2017; 50: 1700648.

Dheda K, Migliori GB. The global rise of extensively drug-resistant tuberculosis: is the time to bring back sanatoria now overdue? Lancet 2012; 379: 773-775.

Fitzpatrick C, Floyd K. A systematic review of the cost and cost effectiveness of treatment for multidrug-resistant tuberculosis. Pharmacoeconomics 2012; 30: 63-80.

Cox H, Hughes J, Daniels J, et al. Community-based treatment of drug-resistant tuberculosis in Khayelitsha, South Africa. Int J Tuberc Lung Dis 2014; 18: 441-448.

Cox HS, Furin JJ, Mitnick CD, et al. The need to accelerate access to new drugs for multidrug-resistant tuberculosis. Bull World Health Organ 2015; 93: 491-497.

Loveday M, Wallengren K, Brust J, et al. Community-based care vs. centralized hospitalization for MDR-TB patients, KwaZulu-Natal, South Africa. Int J Tuberc Lung Dis 2015; 19: 163-171.

Malla P, Kanitz EE, Akhtar M, et al. Ambulatory-based standardized therapy for multidrug-resistant tuberculosis: experience from Nepal, 2005-2006. PLoS One 2009; 4: e8313

Bureau of Tuberculosis Control. Tuberculosis Clinical Policies and Protocols, 4th Edn. New York, Dept of Health and Mental Hygiene, 2008.

Weiss P, Chen W, Cook VJ, et al. Treatment outcomes from community-based drug resistant tuberculosis treatment programs: a systematic review and meta-analysis. BMC Infect Dis 2014; 14: 333.

Farley JE, Kelly AM, Reiser K, et al. Development and evaluation of a pilot nurse case management model to address multidrug-resistant tuberculosis (MDR-TB) and HIV in South Africa. PLoS One 2014; 9: e111702.

Horne DJ, Johnson CO, Oren E, et al. How soon should patients with smear-positive tuberculosis be released from inpatient isolation? Infect Control Hosp Epidemiol 2010; 31: 78-84. 
Andrews RH, Devadatta S, Fox W, et al. Prevalence of tuberculosis among close family contacts of tuberculous patients in South India, and influence of segregation of the patient on early attack rate. Bull World Health Organ 1960; 23: 463-510.

117 Crofton J. The contribution of treatment to the prevention of tuberculosis. Bull Int Union Tuberc 1962; 32: 643-653.

118 Schwartzman K, Menzies D. Tuberculosis: 11. Nosocomial disease. CMAJ 1999; 161: 1271-1277.

119 Petersen E, Khamis F, Migliori GB, et al. De-isolation of patients with pulmonary tuberculosis after start of treatment - clear, unequivocal guidelines are missing. Int J Infect Dis 2017; 56: 34-38.

120 Bassili A, Fitzpatrick C, Qadeer E, et al. A systematic review of the effectiveness of hospital- and ambulatory-based management of multidrug-resistant tuberculosis. Am J Trop Med Hyg 2013; 89: 271-280.

121 Simonovska L, Ilievska-Popovska B. Comparison of results from inpatient and outpatient treatment of tuberculosis in Republic of Macedonia. Open Access Maced J Med Sci 2015; 3: 337-340.

122 Horter S, Stringer B, Reynolds L, et al. "Home is where the patient is": a qualitative analysis of a patient-centred model of care for multidrug-resistant tuberculosis. BMC Health Serv Res 2014; 14: 81.

123 Padayatchi N, Friedland G. Decentralized management of drug-resistant tuberculosis (MDR- and XDR-TB) in South Africa: an alternative model of care. Int J Tuberc Lung Dis 2008; 12: 978-980.

124 van Cutsem G, Isaakidis P, Farley J, et al. Infection control for drug-resistant tuberculosis: early diagnosis and treatment is the key. Clin Infect Dis 2016; 62: Suppl. 3, S238-S243.

125 Yuen CM, Amanullah F, Dharmadhikari A, et al. Turning off the tap: stopping tuberculosis transmission through active case-finding and prompt effective treatment. Lancet 2015; 386: 2334-2343.

126 Jenkins HE, Crudu V, Soltan V, et al. High risk and rapid appearance of multidrug resistance during tuberculosis treatment in Moldova. Eur Respir J 2014; 43: 1132-1141.

127 Atun RA, Samyshkin YA, Drobniewski F, et al. Social factors influencing hospital utilization by tuberculosis patients in the Russian Federation: analysis of routinely collected data. Int J Tuberc Lung Dis 2005; 9: 1140-1146.

128 Nardell EA, Brickner PW. Tuberculosis in New York City: focal transmission of an often fatal disease. JAMA 1996; 276: 1259-1260.

129 Nutini S, Fiorenti F, Codecasa LR, et al. Hospital admission policy for tuberculosis in pulmonary centres in Italy: a national survey. AIPO Tuberculosis Study Group Italian Association of Hospital Pulmonologists. Int J Tuberc Lung Dis 1999; 3: 985-991.

130 Atun RA, Baeza J, Drobniewski F, et al. Implementing WHO DOTS strategy in the Russian Federation: stakeholder attitudes. Health Policy 2005; 74: 122-132.

131 Gandhi NR, Nunn P, Dheda K, et al. Multidrug-resistant and extensively drug-resistant tuberculosis: a threat to global control of tuberculosis. Lancet 2010; 375: 1830-1843. 